\title{
A REMARK ON THE BOSON-FERMION CORRESPONDENCE
}

\author{
Yurii A. Neretin \$ \\ Chair of Mathematical Analysis, Moscow Inst. of Electronics and Math- \\ ematics Bol'shoi Triohsviatitel'skii per. 3/12, Moscow 109082, Russia, \\ E-mail: chuhloma@neretin.mccme.ru
}

\begin{abstract}
We introduce the space of skew-symmetric functions depending on an infinite number of variables and give a simple interpretation of the boson-fermion correspondence.
\end{abstract}

The boson-fermion correspondence (Skyrme, 1971) is a canonical transformation from bosonic Fock space to fermionic Fock space (more precisely it is an operator from some special bosonic Fock space to some special fermionic Fock space). Now it is a quite well-known object in mathematics and mathematical physics (see for instance [3]). The purpose of this note is to give a very simple description of this operator. The bosonfermion correspondence will be multiplication with the Vandermonde determinant. In some sense our description is not new (it is equivalent to an explanation which uses Schur functions, see [3]), on the other side I never have seen this description in the literature and never have heard about it.

1. Bosonic Fock space F. Consider formal variables $z_{1}, z_{2}, \ldots$. Consider the space $P o l$ of polynomials in the variables $z_{1}, z_{2}, \ldots$. Define a scalar product in $P o l$ by the following rule: the monomials $z_{1}^{k_{1}} z_{2}^{k_{2}} \ldots$ are pairwise orthogonal and

$$
\left\|z_{1}^{k_{1}} z_{2}^{k_{2}} \ldots\right\|^{2}=\prod_{j}\left(k_{j} ! j^{k_{j}}\right)
$$

We define the bosonic Fock space $\mathbf{F}$ (V.A.Fock, 1929, see [3, 2]) as the completion of Pol with respect to this scalar product.

2. Space Symm of symmetric functions. Consider an infinite collection of formal variables $x_{1}, x_{2}, \ldots$. We define the space Symm of symmetric functions as the space of symmetric infinite formal sums of monomials in the variables $x_{1}, x_{2}, \ldots$ (see [1]) (in each monomial only finite number of variables occurs).

Denote by $p_{k}$ the infinite Newton sums

$$
p_{n}=x_{1}^{n}+x_{2}^{n}+x_{3}^{n}+\ldots
$$

\footnotetext{
${ }^{1}$ supported by RFBR grant 98-01-00303 and by Russian program of support of scientific schools
} 
The classical scalar product (J.H. Redfield, 1927) in the space Symm is given by the rule: the "functions" $p_{1}^{k_{1}} p_{2}^{k_{2}} \ldots$ are orthogonal and

$$
\left\|p_{1}^{k_{1}} p_{2}^{k_{2}} \ldots\right\|^{2}=\prod_{j}\left(k_{j} ! j^{k_{j}}\right)
$$

3. Boson-symmetric correspondence, see [3]. A canonical isometry $I: \mathbf{F} \rightarrow$ Symm is given by the rule

$$
I: z_{1}^{k_{1}} z_{2}^{k_{2}} \ldots \mapsto p_{1}^{k_{1}} p_{2}^{k_{2}} \ldots
$$

In other words the operator $I$ is a substitution operator

$$
I f\left(x_{1}, x_{2}, x_{3}, \ldots\right)=f\left(\sum_{j} x_{j}, \sum_{j} x_{j}^{2}, \sum_{j} x_{j}^{3}, \ldots\right) .
$$

Obviously $I$ is an isometry (see (1) and (2)).

4. Space of skew-symmetric functions. This object is very simple but psychologically strange. Consider the same variables $x_{1}, x_{2}, \ldots$ A quasi-monomial is a formal expression

$$
x_{1}^{\omega+l_{1}} x_{2}^{\omega+l_{2}} x_{3}^{\omega+l_{3}} \ldots
$$

where $l_{j}=-j$ for large $j$ and $\omega$ is a formal symbol. A skew-symmetric function is a formal (infinite) linear combination of quasi-monomials which is skew-symmetric with respect to all finite permutations of the variables $x_{1}, x_{2}, \ldots$

REMARK. Informally, $\omega$ means

$$
\omega=\infty .
$$

It is "the total number" of variables $x_{1}, x_{2}, \ldots$ It is natural to consider the expression

$$
\prod_{1 \leq i<j<\infty}\left(x_{i}-x_{j}\right)
$$

as skew-symmetric function. Indeed let us write this expression in the form

$$
\prod_{1 \leq i<j<\infty}\left\{x_{i}\left(1-\frac{x_{j}}{x_{i}}\right)\right\}=\prod_{1 \leq i<j<\infty} x_{i} \prod_{1 \leq i<j<\infty}\left(1-\frac{x_{j}}{x_{i}}\right)
$$

We obtain

$$
\prod_{1 \leq i<j<\infty}\left(x_{i}-x_{j}\right)=\sum_{\sigma \in S_{\infty}}(-1)^{\sigma} x_{1}^{\omega-\sigma(1)} x_{2}^{\omega-\sigma(2)} \cdots
$$

where $S_{\infty}$ is the group of all finite permutations of the set $\{1,2,3,4, \ldots\}$.

Let $l_{1}<l_{2}<l_{3}<\ldots$ be integers and let $l_{j}=j$ for large $j$. Consider the basic skew-symmetric functions

$$
S_{l_{1}, l_{2}, \ldots}=\sum_{\sigma \in S_{\infty}}(-1)^{\sigma} x_{1}^{\omega-l_{\sigma(1)}} x_{2}^{\omega-l_{\sigma(2)}} x_{3}^{\omega-l_{\sigma}(3)}
$$

A scalar product in the space Asymm of skew-symmetric functions is defined by the rule: the functions $S_{l_{1}, l_{2}, \ldots}$ form an orthonormal basis in Asymm.

5. Correspondence between Symm and Asymm. A canonical isometry $J: \mathbf{S y m m} \rightarrow$ Asymm is given by the formula

$$
J f\left(x_{1}, x_{2}, \ldots\right)=f\left(x_{1}, x_{2}, \ldots\right) \cdot \prod_{1 \leq i<j<\infty}\left(x_{i}-x_{j}\right)
$$


6. Fermionic Fock space, see [3, 2]. Let $\ldots \xi_{-2}, \xi_{-1}, \xi_{0}, \xi_{1}, \xi_{2}, \ldots$ be a family of anticommuting variables $\left(\xi_{i} \xi_{j}=-\xi_{j} \xi_{i}\right)$. Consider infinite products

$$
\xi_{l_{1}} \xi_{l_{2}} \xi_{l_{3}} \ldots
$$

where $l_{1}<l_{2}<\ldots$ and $l_{j}=j$ for large $j$. We define the fermionic Fock space $\boldsymbol{\Lambda}$ as the space where the monomials (5) form an orthonormal basis.

7. Isometry between Symm and $\boldsymbol{\Lambda}$. This correspondence is obvious: the basis element (4) corresponds to the basis element (5).

8. The boson-fermion correspondence is the composition of the correspondences

$$
\mathrm{F} \rightarrow \text { Symm } \rightarrow \text { Asymm } \rightarrow \Lambda
$$

In fact it is the composition of the substitution

$$
z_{k}=\sum_{j} x_{j}^{k}
$$

and the multiplication with the Vandermonde determinant (3).

Some additionsl discussion of boson-symmetric correspondences is contained in [4], [5]

\section{References}

[1] Macdonald I.G., Symmetric functions. Second edition. Clarendon Press, Oxford, 1995.

[2] Neretin Yu.A., Categories of symmetries and infinite dimensional groups. Clarendon Press, Oxford, 1996

[3] Pressley , Segal G., Loop groups. Clarendon Press, Oxford, 1986

[4] Neretin Yu.A., Supercomplete bases in the space of symmetric functions. Funct.Anal.Appl., Vol.32:1, 1998

[5] Neretin Yu.A., On the correspondence between boson Fock space and the $L^{2}$ space over Poisson measure. Sbornik: Mathematics 188:11, 1587-1616 